\title{
Translational Cell Therapy Shared Resource
}

National Cancer Institute

\section{Source}

National Cancer Institute. Translational Cell Therapy Shared Resource. NCI Thesaurus.

Code C39544.

The Translational Cell Therapy Shared Resource serves as a resource for the members of the Cancer Center interested in basic and pre-clinical research involving human cells, developmental therapies and large-scale production of biologic products. The resource provides support in procurement, selection and manipulation of org an tissue, blood or marrow products including stem cells, cell separation, manipulation, and culture, and the development of large-scale processing of cells for clinical trials. 